\title{
PERCEIVED SELF-REGULATORY SUCCESS IN DIETING AND ITS CORRELATES AMONG WOMEN WITH FOOD ADDICTION
}

\author{
Sarah El Archi ${ }^{1}$, Paul Brunault ${ }^{1,2,3}$, Nicolas Ballon ${ }^{2,3}$, Christian Réveillère ${ }^{1}$, \\ \& Servane Barrault ${ }^{1,4}$ \\ ${ }^{1}$ Qualipsy, EE 1901, Université de Tours, 37041 Tours (France) \\ ${ }^{2}$ CHRU de Tours, Service d'Addictologie Universitaire, Équipe de Liaison et de Soins en Addictologie, \\ 37044 Tours (France) \\ ${ }^{3}$ UMR 1253, iBrain, Université de Tours, INSERM, 37032 Tours (France) \\ ${ }^{4}$ CHRU de Tours, Service d'Addictologie Universitaire, Centre de Soins d'Accompagnement \\ et de Prévention en Addictologie d'Indre-et-Loire (CSAPA-37), 37000 Tours (France)
}

\begin{abstract}
Background: Several psychological features are implicated in the dieting success. Better understanding of these features may allow reducing dieting failure of both surgical and non-surgical weight loss interventions, especially for individuals with food addiction (FA). In non-clinical population, low perceived self-regulatory success (PSRS) in dieting is associated with higher BMI (body mass index), FA, food craving and impulsivity. PSRS could partially explain weight gain in FA, but no study investigated this association in the specific FA population. Method: To diagnose FA, 288 women recruited online completed The Yale Food Addiction Scale 2.0. They also completed the following self-administrated questionnaires: the French adaptation of the PSRS in dieting scale, the Food Craving Questionnaire Trait-Reduced, and the Barratt Impulsivity Scale-11. They specified their height, current and lifetime maximal weight, and if they were in a current diet. Mean age was $26.1 \pm 10.3$ years. Mean current BMI was $23.4 \pm 5.5 \mathrm{~kg} / \mathrm{m}^{2}$. Results: 79 women met criteria for FA (27.4\%), indicating significant less PSRS in dieting and higher probability to be in a current diet to lose weight. In the whole population $(n=288)$, PSRS in dieting was negatively correlated with current and lifetime maximal BMI, food craving, FA, attentional and non-planning impulsivity. In a multiple linear regression conducted in the subgroup of women with FA, PSRS score was predicted by age, current BMI, food addiction and food craving. More, results suggested food craving enable the association between food addiction and PSRS. Conclusion: These results showed the high preoccupation about food intake and weight gain in the FA population. Psychological features associated with FA such as food craving and impulsivity, seem to impact the PSRS in dieting, increasing psychological vulnerability.
\end{abstract}

Keywords: Food addiction, perceived self-regulatory success in dieting, food craving, impulsivity, obesity.

\section{Introduction}

To prevent weight gain, individuals with overweight or obesity repeated several periods of dieting to lose weight. But according to Nguyen and Polivy (2014) current dieters are 1.7 times less likely to be considered successful dieters than non-current dieters. More, successful self-regulators had lower body mass index (BMI) than unsuccessful self-regulators. Some disordered eating behavior such as food addiction (FA) symptomatology or binge eating increase difficulty to success in dieting (Meule et al., 2012). Addictive-like eating behavior is indeed associated with higher BMI, higher lifetime BMI, greater likelihood of obesity and regain lost weight (Gearhardt et al., 2016). Development of the Perceived Self-Regulatory Success in Dieting Scale (PSRS) (Fishbach et al., 2003), a self-report of success in dieting, allowed to increase data about perceived success in weight control. Meule and colleagues investigated psychological and eating factors associated with PSRS in dieting. They conducted a study within a population of student women and reported that PSRS was negatively associated with higher BMI, concern for dieting, the use of rigid dieting strategies, food craving, food addiction symptoms, and binge eating frequency. Contrary to what was expected, they highlighted a small negative correlation between PSRS and cognitive impulsivity (Meule et al., 2012). More, they highlighted, through a longitudinal study conducted in female students, that food craving prospectively predicted decreased PSRS in weight regulation six months later (Meule et al., 2017). They suggested a serial mediation model 
with an indirect effect of trait impulsivity on BMI via food cravings and PSRS in dieting (Meule $\&$ Blechert, 2017). Individuals with addictive-like eating behavior who experiment high food cravings struggle to control food consumption and maintain a steady weight. But as far as we know, no study investigated PSRS in dieting within the specific population of individuals with FA symptomatology.

In this way, the main purpose of this study was to investigate the factors associated with PSRS in dieting within a population of individuals with FA symptomatology, including BMI, FA severity, food craving and impulsivity. We expected (1) participants who screen positive for FA would show lower PSRS; (2) PSRS would be negatively correlated and predicted by BMI, FA severity, food craving and impulsivity, with stronger associations for FA participants; (3) FA and food craving is a mediator in the association between PSRS and these variables, even more for FA participants.

\section{Methods}

\subsection{Procedure and participants}

288 women recruited in a non-clinical population filled out the questionnaire, which was put online from January to March 2017. Participants were recruited in French-speaking web forums (varied topics of conversation or especially consecrated to eating behaviors). The questionnaire provided information about socio-demographic characteristics, height, weight, dieting behavior, food addiction, food craving and impulsivity. The Institutional Review Board of the University Hospital of Tours (France) approved this survey, conducted in accordance with the Helsinki Declaration (1989).

\subsection{Measures}

The survey began with some questions about the gender and the age. Participants indicated their current height and weight. More, they indicated their lifetime maximal weight and their ideal body weight. These data allowed to assess the current, maximal and ideal body mass indexes (=BMI), respectively noted $\mathrm{BMI}_{\text {current, }} \mathrm{BMI}_{\max }, \mathrm{BMI}_{\text {ideal, }}$, and allowed calculating the $\mathrm{BMI}_{\text {ideal }}$ rate: $\left(\mathrm{BMI}_{\text {ideal }}-\mathrm{BMI}_{\text {current }}\right) / \mathrm{BMI}_{\text {current }}$. Then, participants answered to the following questions to interrogate their filling of weight control: (1) Are you currently on a diet to control your weight? (2) If your answer is "yes", what is the goal of your diet: to lose or gain weight, or to stabilize your weight? Then, the French adaptation of the perceived self-regulatory success in dieting scale (PSRS) was administered (Fishbach et al., 2003; Meule et al., 2012). The PSRS contains 3 items which interrogate how successful participants are in watching their weight (Cronbach alpha=.70). Higher PSRS scores indicate higher perceived success in controlling their weight.

Finally, the questionnaire involved filling out the French version of the following scales:

The Food Craving Questionnaire-Trait reduced (FCQ-T-r) is composed of 15 items (Brunault et al., 2018; Cepeda-Benito et al., 2000; Meule et al., 2014). This scale questions about food craving and preoccupations with food (Cronbach alpha: .94). Participants answered on a 6-points Likert scale from "1-never" to "6-always". The sum of the item scores provides the FCQ-T-r score, which increases with food craving severity.

The Yale Food Addiction Scale 2.0 (YFAS 2.0) assess food addiction and consists of 35 items questioning last 12 months eating behaviors (Brunault et al., 2017; Gearhardt et al., 2016). Each item refers to one of the 11 criteria of addictive-like eating behavior, by extrapolating to food the DSM-5 criteria for addictive disorders. Participants indicated on a 7 points Likert scale the frequency of this symptomatology (from "0-Never" to "7-Every day"). YFAS 2.0 provides 2 types of data: self-reported FA (at least 2 criteria and clinically significant distress or impairment) (Cronbach alpha=.97) and a total score indicating the number of FA symptoms.

The Barratt Impulsiveness Scale 11 (BIS-11) consists of 30 items assessing 3 impulsivity dimensions: cognitive (8 items, Cronbach alpha: .64), motor (11 items, Cronbach alpha: .65) and non-planning (11 items, Cronbach alpha: .65) (Balbinotti et al., 2015; Barratt, 1959). For each item, participants filled out a 4-points Likert scale scored from "almost never/never" to "almost always/always". BIS-11 scores increase with the impulsivity severity.

\subsection{Data analysis}

Results on the YFAS 2.0 allowed distinguishing individuals with FA from other participants. Logistic regression analyses assessed difference in terms of PSRS in dieting, $\mathrm{BMI}_{\text {current }}, \mathrm{BMI}_{\max }, \mathrm{BMI}_{\text {ideal }}$ rate and current dieting between FA and non-FA participants. Spearman correlation highlighted factors associated with PSRS in dieting and partial correlations investigated the impact of control variables such as YFAS 2.0 and FCQ-T-r. Finally, simple and multiple linear regression analyses assessed the mediator role of food craving between PSRS and FA, the mediator role of FA between PSRS and impulsivity and identified the predicted factors of PSRS. All analyses were two-tailed and considered $\mathrm{p} \leq .05$ as statistically significant. 


\section{Results}

\subsection{Sample description}

The mean age of the participants was $26.1 \pm 10.3$ years old. Seventy-nine participants $(27.4 \%$ of the whole sample) met criteria for self-reported FA. Mean BMI $\mathrm{Burrent}_{\text {t }}$ was $23.4 \pm 5.5 \mathrm{~kg} / \mathrm{m}^{2}$ corresponding to normal weight. Mean $\mathrm{BMI}_{\max }$ was $25.7 \pm 6.8 \mathrm{~kg} / \mathrm{m}^{2}$ corresponding to overweight. The mean $\mathrm{BMI}_{\text {ideal }}$ rate was $-10.3 \pm 10.4 \%$. So then, on average, participants estimated their current weight $10.3 \%$ higher that the ideal one. $40.6 \%$ of the whole sample was in a current diet to lose weight.

\subsection{Factors associated with PSRS in dieting in the whole sample}

PSRS in dieting was significantly negatively correlated with current, maximal BMI, FCQ-T-r, YFAS 2.0 and positively correlated with the $\mathrm{BMI}_{\text {ideal }}$ rate (details in Table 1). Moreover, there are slightly negative correlation between PSRS and cognitive and non-planning impulsivity. If YFAS 2.0 or FCQ-T-r was controlled, correlation between PSRS, current, maximal BMI, and the $\mathrm{BMI}_{\text {ideal }}$ rate were slightly lower and still significant, but correlation with BIS-11 was no longer significant (Table 1). It is notable that FCQ-T-r controlled, correlation between PSRS in dieting and YFAS 2.0 disappeared.

The linear regression analyses suggested food craving enable the association between FA and PSRS (in a simple linear regression, food craving was predicted with $F A$ : $B=3.77, t=14.37, p<.001$; in a multiple linear regression, PSRS was predicted with food craving $(B=-.09, \mathrm{t}=-.37, \mathrm{p}<.001)$, not with FA $(\mathrm{B}=-.04, \mathrm{t}=-.45, \mathrm{p}=.65))$. And FA enable the association between PSRS and cognitive (FA was predicted with cognitive BIS-11: $\mathrm{B}=.30, \mathrm{t}=6.29, \mathrm{p}<.001$; PSRS was predicted with $\mathrm{FA}(\mathrm{B}=-.35, \mathrm{t}=-4.78, \mathrm{p}<.001)$, not with cognitive BIS-11 ( $\mathrm{B}=-.04, \mathrm{t}=-.69, \mathrm{p}=.49)$ and motor impulsivity (FA was predicted with motor BIS-11: $\mathrm{B}=.30, \mathrm{t}=6.29, \mathrm{p}<.001$; PSRS was predicted with $\mathrm{FA}(\mathrm{B}=-.38, \mathrm{t}=-5.23, \mathrm{p}<.001)$, not with motor BIS-11 $(\mathrm{B}=.02, \mathrm{t}=.32, \mathrm{p}=.75)$. More the variance of PSRS in dieting was explaining by the following variables: $\mathrm{BMI}_{\text {current }}, \mathrm{FCQ}-\mathrm{T}-\mathrm{r}$, BIS-non planning and BIS-motor $\left(\mathrm{n}=288, \mathrm{R}^{2}=.319\right.$. $\mathrm{F}(4,283)=33.168$, $\mathrm{p}<.001)$. Details in Table $2 \mathrm{a}$.

Table 1. Spearman correlations between PSRS in dieting and various variables.

\begin{tabular}{|c|c|c|c|c|c|c|c|c|c|c|c|c|}
\hline \multirow[b]{3}{*}{ Control variables } & \multicolumn{6}{|c|}{ All participants $(n=288)$} & \multicolumn{6}{|c|}{ Participants with FA $(n=79)$} \\
\hline & \multicolumn{6}{|c|}{ PSRS correlation } & \multicolumn{6}{|c|}{ PSRS correlation } \\
\hline & \multicolumn{2}{|c|}{ None } & \multicolumn{2}{|c|}{ YFAS 2.0} & \multicolumn{2}{|c|}{ FCQ-T-r } & \multicolumn{2}{|c|}{ None } & \multicolumn{2}{|c|}{ YFAS 2.0} & \multicolumn{2}{|c|}{ FCQ-T-r } \\
\hline Current BMI & -.445 & $* * *$ & -.416 & $* * *$ & -.394 & $* * *$ & -.578 & $* * *$ & -.524 & $* * *$ & -.431 & $* * *$ \\
\hline Maximal BMI & -.339 & $* * *$ & -.252 & $* * *$ & -.220 & $* * *$ & -.373 & $* *$ & -.256 & $* * *$ & -.151 & $* * *$ \\
\hline BMIIDEAL rate & .450 & $* * *$ & .392 & $* * *$ & .370 & $* * *$ & .420 & $* * *$ & .417 & $* * *$ & .347 & $* * *$ \\
\hline YFAS 2.0 & -.294 & $* * *$ & - & & -.027 & & -.086 & & - & & -.200 & \\
\hline FCQ-T-r & -.370 & $* * *$ & -.273 & $* * *$ & - & & -.407 & $* * *$ & -.451 & $* * *$ & - & \\
\hline BIS-11 Total & -.129 & $*$ & -.064 & & -.024 & & -.137 & & -.131 & & -.025 & \\
\hline BIS-11 Cognitive & -.137 & $*$ & -.041 & & -.009 & & -.156 & & -.132 & & -.041 & \\
\hline BIS-11 Motor & -.023 & & .019 & & .072 & & -.149 & & .185 & & .071 & \\
\hline BIS-11 Non-planning & -.120 & $*$ & -.120 & $*$ & -.106 & & -.009 & & -.006 & & -.049 & \\
\hline
\end{tabular}

\subsection{Factors associated with PSRS in dieting in the sample participants with FA}

Logistic regression analyses showed that FA participants had lower PSRS mean score $(9.15 \pm 3.75$ vs $11.37 \pm 3.90$, odds ratio $(\mathrm{OR})=.86,95 \%$ confidence interval $(\mathrm{CI}): .80-.92, \mathrm{p}<.001)$ than non-FA participants. They had higher current and maximal BMI (OR=1.07, 95\% CI: 1.02-1.12, $\mathrm{p}=.006$ and $\mathrm{OR}=1.11,95 \% \mathrm{CI}: 1.06-1.16, \mathrm{p}<.001$ respectively) and lower $\mathrm{BMI}_{\text {ideal }}$ rate $(\mathrm{OR}=.94,95 \% \mathrm{CI}: .91-.96$, $\mathrm{p}<.001$ ). Moreover, $60.8 \%$ and $33 \%$ respectively of the FA participants and non-FA participants declared be in a current diet to lose weight $(\mathrm{p}<.001)$. As for the whole sample, PSRS was correlated with $\mathrm{BMI}_{\text {current, }}$ $\mathrm{BMI}_{\max }, \mathrm{BMI}_{\text {ideal }}$ rate, and FCQ-T-r. It should be noted that correlation coefficients are higher in this group. But there was no correlation with YFAS 2.0 and BIS-11. YFAS 2.0 or FCQ-T-r controlled, correlation between PSRS and $\mathrm{BMI}_{\text {current }}$ and $\mathrm{BMI}_{\max }$ were slightly lower. Moreover YFAS 2.0 controlled, correlation between PSRS and FCQ-T-r increase. Details in Table 1.

The linear regression analyses conducted with FA participants suggested food craving enable the association between FA and PSRS (in a simple linear regression, food craving was predicted with FA: $B=3.03, t=5.79, p<.001$; in a multiple linear regression, PSRS was predicted with food craving $(\mathrm{B}=-.14, \mathrm{t}=-4.41, \mathrm{p}<.001)$, not with FA $(\mathrm{B}=-.31, \mathrm{t}=1.78, \mathrm{p}=.08))$. But contrary to the whole sample, FA 
did not enable the association between PSRS and impulsivity. The multiple linear regression highlighted

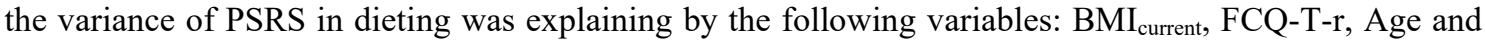
YFAS $2.0\left(n=79, R^{2}=.397 . F(4,78)=12.192, p<.001\right)($ Table $2 b)$.

Table 2a. Variables explaining the variance of PSRS through multiple linear regression in the whole participants $(n=288)$.

\begin{tabular}{lccc}
\hline & B & t & p \\
\hline Constant & 21.806 & 15.355 & $<.001$ \\
BMICURRENT & -.277 & -7.559 & $<.001$ \\
FCQ-T-r & -.075 & -6.010 & $<.001$ \\
BIS-11 non-planning & -.152 & -3.292 & .001 \\
BIS-11 motor & .133 & 2.568 & .011
\end{tabular}

Table 2b. Variables explaining the variance of PSRS through multiple linear regression in FA participants

$$
(n=79) \text {. }
$$

\begin{tabular}{lccc}
\hline & B & t & p \\
\hline Constant & 20.791 & 10.727 & $<.001$ \\
BMICURRENT & -.196 & -3.907 & $<.001$ \\
FCQ-T-r & -.109 & -3.498 & .001 \\
Age & -.068 & -2.227 & .029 \\
YFAS 2.0 & .315 & 2.020 & .047
\end{tabular}

Note. PSRS: Perceived Stress Regulatory Success in dieting scale; BMI: Body Mass Index; FCQ-T-r: Food Craving Questionnaire Trait reduced; YFAS 2.0: Yale Food Addiction Scale 2.0; BIS-11: Barratt Impulsiveness Scale 11.

\section{Discussion}

The purpose of this study was to investigate the factors associated with perceived self-regulatory success in dieting, especially within a population of individuals with and without FA.

In the group of individuals with FA, PSRS in dieting and the $\mathrm{BMI}_{\text {ideal }}$ rate were lower, and the rate of individuals in a current dieting to lose weight was higher, suggesting specific difficulties to control body mass and many preoccupations about food intake and weight gain. Rate of FA among population of individuals with DSM-5 eating disorders is high (Fauconnier et al., 2020). Anorexia nervosa involves restriction of energy intake and intense fear of gaining weight, bulimia nervosa and binge eating disorder involve recurrent episodes of binge eating followed by compensatory behaviors or not. Thus, in these populations, preoccupations with food intake and weight gain, and resort to episode of dieting are high, explaining our results highlighted higher dieting rate, and lower PSRS in dieting and $\mathrm{BMI}_{\text {ideal }}$ rate in individuals with FA symptomatology. When FA involve episodes of overeating not associated with compensatory behaviors, it may lead to weight gain and obesity. Faced with many energy-dense food intakes dieting is an attempt to control negative repercussion of binge eating. Successful weight loss is more difficult for FA individuals because they usually have higher BMI (Gearhardt et al., 2016), more weight to lose, ideal weight very lower than current weight and FA involves severe struggle to control food intakes which increase difficulties to success in dieting. Without clinical care, dieting episodes are rarely successful in the long term. Dieting failures are high and increase difficulties with body mass control. The alternation of food intake deprivation and overconsumption causes disordered eating behaviors, lead to an addictive process, and considerably impact self-esteem.

As expected, among the whole sample, PSRS was negatively correlated with current and maximal lifetime BMI, food craving, food addiction and cognitive and non-planning impulsivity. These results are in line with the study of Meule et al. (2012), who suggested that PSRS in dieting is especially correlated with factors of self-regulatory failure in eating behavior. However, contrary to their study, correlation coefficients were slightly lower for the present study and we highlighted small negative correlation between PSRS and non-planning impulsivity. PSRS coefficient correlations with BMI and food craving increased when we considered only participants with FA self-administered diagnosis. But contrary to what was expected, correlation with FA and impulsivity disappeared. However, in this group, PSRS in dieting was predictable with current BMI, food craving, age, and food addiction severity. Results also suggested a mediator role of food craving between PSRS and FA, and a mediator role of FA between the association of PSRS and impulsivity, not reported by FA participants. According to these results, psychological interventions could target food craving to impact perceived body mass control, which could be further investigated by future studies. Current investigations are in line with the serial mediation model of Meule and Blechert (2017), suggesting an indirect effect of impulsivity on BMI via food craving and PSRS in dieting. Moreover, according to our results, we hypothesized food addiction is an additional factor which could be a mediator between impulsivity and food craving. But these results were not confirmed for individuals with FA symptomatology.

Because of some limitations, these results should be cautiously interpretated. Firstly, this study provides results from cross sectional design. That do not make it possible to establish causal links. Moreover, participants were recruited online, and all collected data were self-reported. We did not have any way to check information. Further study should plan clinical interview to verify the height, weight and FA diagnose. That would enable collecting reliable data. Finally, food addiction is still under debate. 
There is no clear consensus on the concept of food addiction. Some authors are skeptical of the substance use disorder criteria translation to food intake, and question the pharmacological effect of food on the brain (Fletcher \& Kenny, 2018). However, individuals with FA reported similar symptomatology such as craving, distress, difficulty to control their behavior and overconsumption despite negative repercussion (on health, sociability...). More, regarding the variety of eating disorders associated with food addiction is still difficult to provide a consistent vision of FA population (Fauconnier et al., 2020).

Although this study has some limitations, it provides several data about how individuals with FA symptomatology success in watching their weight and associated factors. We may suggest the key role of food craving associated with low PSRS in dieting involving weight gain and distress. Further studies should investigate the serial mediation model within FA clinical population and opt for longitudinal design providing causal links.

\section{References}

Balbinotti, M. A. A., Gélinas, S., \& Labonté, S.-H. (2015). Factor Analysis of French Translation of the Barratt Impulsiveness Scale (BIS-11). Saúde e Desenvolvimento Humano, 3(2). https://doi.org/10.18316/2317-8582.15.5

Barratt, E. S. (1959). Anxiety and Impulsiveness Related to Psychomotor Efficiency. Perceptual and Motor Skills, 9(3), 191-198. https://doi.org/10.2466/pms.1959.9.3.191

Brunault, P., Courtois, R., Gearhardt, A. N., Gaillard, P., Journiac, K., Cathelain, S., Réveillère, C., \& Ballon, N. (2017). Validation of the French Version of the DSM-5 Yale Food Addiction Scale in a Nonclinical Sample. Canadian Journal of Psychiatry, 62(3), 199-210. https://doi.org/10.1177/0706743716673320

Brunault, P., El Archi, S., Ballon, N., Réveillère, C., \& Barrault, S. (2018). Validation of the French version of the Food Cravings Questionaire-Trait-reduced: An easy-to-use and quick self-administered questionnaire to assess food craving. Annales Medico-Psychologiques, 176(8), 788-795. https://doi.org/10.1016/j.amp.2018.08.002

Cepeda-Benito, A., Gleaves, D. H., Williams, T. L., \& Erath, S. A. (2000). The development and validation of the state and trait food-cravings questionnaires. Behavior Therapy, 31(1), 151-173. https://doi.org/10.1016/S0005-7894(00)80009-X

Fauconnier, M., Rousselet, M., Brunault, P., Thiabaud, E., Lambert, S., Rocher, B., Challet-Bouju, G., \& Grall-Bronnec, M. (2020). Food Addiction among Female Patients Seeking Treatment for an Eating Disorder: Prevalence and Associated Factors. Nutrients, 12(6), 1897. https://doi.org/10.3390/nu12061897

Fishbach, A., Friedman, R. S., \& Kruglanski, A. W. (2003). Leading is not unto Temptation: Momentary Allurements Elicit Overriding Goal Activation. Journal of Personality and Social Psychology, 84(2), 296-309. https://doi.org/10.1037/0022-3514.84.2.296

Fletcher, P. C., \& Kenny, P. J. (2018). Food addiction: a valid concept? Neuropsychopharmacology, 43(13), 2506-2513. https://doi.org/10.1038/s41386-018-0203-9

Gearhardt, A. N., Corbin, W. R., \& Brownell, K. D. (2016). Supplemental Material for Development of the Yale Food Addiction Scale Version 2.0. Psychology of Addictive Behaviors, 30(1), 113-121. https://doi.org/10.1037/adb0000136.supp

Meule, A., \& Blechert, J. (2017). Indirect effects of trait impulsivity on body mass. Eating Behaviors, 26, 66-69. https://doi.org/10.1016/j.eatbeh.2017.01.012

Meule, A., Hermann, T., \& Kübler, A. (2014). A short version of the food cravings questionnaire-trait: The FCQ-T-reduced. Frontiers in Psychology, 5(MAR). https://doi.org/10.3389/fpsyg.2014.00190

Meule, A., Papies, E. K., \& Kübler, A. (2012). Differentiating between successful and unsuccessful dieters. Validity and reliability of the Perceived Self-Regulatory Success in Dieting Scale. Appetite, 58(3), 822-826. https://doi.org/10.1016/j.appet.2012.01.028

Meule, A., Richard, A., \& Platte, P. (2017). Food cravings prospectively predict decreases in perceived self-regulatory success in dieting. Eating Behaviors, 24, 34-38. https://doi.org/10.1016/j.eatbeh.2016.11.007

Nguyen, C., \& Polivy, J. (2014). Eating behavior, restraint status, and BMI of individuals high and low in perceived self-regulatory success. Appetite, 75, 49-53. https://doi.org/10.1016/j.appet.2013.12.016 